\title{
RSVP: A task, reading aid, and research tool
}

\author{
SHERYL R. YOUNG \\ University of Colorado, Boulder, Colorado
}

\begin{abstract}
Computer presentation of text material is evaluated with respect to its efficiency relative to normal reading. All results are examined in light of the various task analyses associated with different variations on the technique. Finally, the utility of computer presentation as a research tool for investigating human information processing is addressed.
\end{abstract}

Rapid serial visual presentation (RSVP) is a technique that has been used to present textual information to readers on a CRT screen. This paper is designed to examine what RSVP is, how it is being utilized, and what effects it can have.

The first part of this discussion addresses the various operational definitions of RSVP and attempts to present a task analysis of the technique by delineating its differences from normal reading and examining them with respect to information processing implications. Second, a brief outline of the requirements for setting up a system to present text via RSVP is presented. The last section addresses the practical use of RSVP to increase reading efficiency and improve reading comprehension, as well as its role as a research tool.

\section{WHAT IS RSVP?}

RSVP is a term initially coined by Forster (1970), who used it to refer to presenting, at rapid rates, single words to a stationary position in the viewing field. Although this single-word-at-a-time notion has persisted, more recently other unit sizes have been explored. For example, Juola, Ward, and McNamara (1982) varied the number of characters presented in a stationary window from 5 to 15 , with the constraint that complete words were always presented. Young, Angell, and Bourne (1984) manipulated unit size by using clauses, single words, three words, and sentences as the units of input. Thus, the operational definition of RSVP varies considerably.

Another variation on the RSVP technique is to present small amounts of text to the reader on each viewing, but to locate the successive units such that the text is selectively unblanked in the position it would normally occupy if the entire text were displayed on the CRT screen. This variant will hereafter be referred to as "moving RSVP." An example of moving RSVP was described by Just, Carpenter, and Woolley (1982), who used a single-word unit size but did not adhere to the stationary convention.

Given the ways in which RSVP has been used, the

The author's mailing address is: Institute of Cognitive Science, Muenzinger Psychology Building. University of Colorado, Boulder, CO 80309 following characterization could serve as a general operational definition of RSVP: RSVP involves successive presentation of small units of text to a viewer. The units of text usually do not exceed a single line and are normally presented for a preselected amount of time.

\section{HOW RSVP DIFFERS FROM NORMAL READING: A TASK ANALYSIS}

Although the manner in which the RSVP technique has been employed varies considerably across investigations, there are always differences between RSVP and normal reading. These differences and the task variations encompassed by them have many implications, which will be briefly addressed.

One consequence of using RSVP to present text is that people see all parts of a text. This point was demonstrated by Potter, Kroll, and Harris (1980) when they had people read normally and via RSVP at 240, 480, and $720 \mathrm{words} / \mathrm{min}$. They found that the people reading normally recalled significantly less information from the second half of the text than did the RSVP readers at reading rates faster than 240 words/min.

When RSVP is used to present units of text to a stationary location in the viewing field (stationary RSVP), eye movements tend to be minimized, especially if a single-word unit size is employed. Even when more than one word at a time is presented, it is impossible to skip lines of text, read words from more than one line at a time, or read the second word in a line and then regress to the first (Just \& Carpenter, 1980).

\section{Several Factors Influence the Reader's \\ Performance Under RSVP}

It should be clear that thorough task analyses are a function of the specific ways in which RSVP is implemented. For example, each unit size used in RSVP has different information processing implications. If one uses a unit size of three words at a time, irrespective of clausal and sentence boundaries, it is most likely that chunking operations may be interfered with. Whether moving or stationary RSVP is used to present units of text has implications for the eye movement system as well as for the unit size employed. Another important distinction with stationary presentation of information 
is whether the information to be presented is centered on a certain area of the CRT screen or whether the leftmost position is held constant. Certainly, less predictability would be associated with the former. The main point is that RSVP task analyses vary as a function of unit size and whether text is presented in a stationary position on the CRT or the text "moves" across the screen. If text is presented in a stationary position, much of the processing time associated with eye movement execution, planning, and inaccuracies is eliminated.

\section{TASK ANALYSES OF SELECTED RSVP TECHNIQUES}

Since a single gaze is executed when text is presented a single word at a time to a stationary position in the viewing field, eye movements, their associated inaccuracies, and hypothesized resource demands are radically reduced (Just \& Carpenter, 1980). Additionally, the reader knows exactly where to direct his/her gaze. Consequently, the need to devote attention to planning the next gaze is eliminated. All one has to do is to recognize and process the input information. Another way single-word stationary presentation of text alters the reading task is that the reader views every word. Thus, words that might normally be skipped or skimmed are viewed. A third consequence of using single-word stationary RSVP is that each word is visible for exactly the same amount of time. A few investigators have expressed concern over the effects of fixed wordexposure time on comprehension, citing the fact that, in normal reading, gaze durations vary across words (Just et al., 1982). Such concerns prompted a recent study by Ward (1981), who demonstrated that reading comprehension does not decrement as a result of having words visible for a constant amount of time as opposed to variable amounts of time normally spent on individual words. Furthermore, the effect of viewing every word appears to improve comprehension in later portions of a text relative to normal reading when rapid rates of input are used (Potter et al., 1980).

When very rapid rates of presentation are used in conjunction with single-word stationary RSVP, and pauses are not inserted at sentence boundaries, chunk. ing and integration may be impaired. RSVP techniques result in comprehenison that is inferior to normal skim. ming at 600 words/min when pauses are not inserted at the ends of sentences (Masson, 1983). Insertion of pauses eliminates the decrement in comprehension associated with RSVP presentation. Thus, although single-word unit size and stationary presentation radically reduce eye movement requirements, they also can interfere with some component processing, if care is not taken to ensure that they do not. The current estimate of word-recognition time is approximately $50 \mathrm{msec}$, whereas average gaze duration is $250 \mathrm{msec}$ (Carr, 1981). Word recognition is only one of the pro- cesses normally executed during a fixation. There are many additional component processes involved in reading that may or may not be executed during a fixation, including chunking, integration, inference making, and connecting incoming information with world knowledge. Masson's results indicate that single-word stationary RSVP may, at rapid rates, interfere with some of these other operations.

What happens when clause-sized units of text are presented to the reader at speeded rates? Our data (also see Cocklin, Ward, Chen, \& Juola, 1984) indicate that RSVP is superior to normal reading. Why? What is involved in this task? First, although eye movements aren't eliminated-certainly more than one gaze is executed when a number of words are presented to the reader-the tendency to read the second word on a line first and then to regress to the first word is restricted by the experimental paradigm. When clauses-stationary RSVP is used to present text, the reader knows exactly where on the screen the first word will appear. Furthermore, the tendency to skip down lines and read a word from one line and the next from a lower line also is eliminated. Thus, the reader must devote a certain amount of effort to execution and planning of eye movements, but they are far more predictable than in the average text.

The second important point concerning clauses is that, theoretically, they approximate the chunks that a reader constructs (Aaronson \& Scarborough, 1977). Thus, chunking requirements are radically reduced, and the reader does not have to determine which words form a coherent meaning unit. This task is performed for the reader by presenting preparsed units of text in the form of meaningful clauses. Thus, although clauses-stationary RSVP does not limit eye movement requirements to the extent that single-word stationary RSVP does, it promotes chunking and thereby reduces the parsing requirements associated with normal reading.

Although both moving and stationary RSVP task analyses are incomplete without a concurrent unitsize task analysis, the moving RSVP task has very different information processing requirements from those of the stationary RSVP task. Moving RSVP requires a subject to continually execute eye movements. However, unlike in normal reading, a subject is prevented from reading one word off line $\mathrm{X}$ and a second word off line $X+1$. Subjects do move their eyes throughout the screen, but the information processing ramifications of this depend on the unit size that is used.

In other words, although brief task analyses of some forms of RSVP have been sketched out, exact task analyses depend upon the specific way in which RSVP is implemented. Later, data are presented that suggest some optimal methods of using RSVP to improve read. ing comprehension. The reasons underlying this improved performance lie in an analysis of the specific information processing requirements of the task. 


\section{SETTING UP A SYSTEM FOR RSVP}

Basically, RSVP can be used on a variety of systems, provided a real-time clock is available. We are currently using a PDP-11 to present text via RSVP and have used a Micro-Nova (Data General Corporation) in the past. Apple II microcomputers have been used by other investigators.

The software designed for running RSVP is as follows. First, the text is preprocessed. Preprocessing involves reading the input text into lists of the unit sizes desired. Additionally, the preprocessing program prints out the number of words in the unit and their starting position (line and column number) in the original unprocessed text. Thus, lists of clauses, three words, individual words, and sentences were produced, each containing the number of words in the unit as well as its position in the original text.

The second stage of the program requests an input of the conditions to be used. We defined moving and stationary conditions for each unit size. The third stage involves querying the experimenter about whether the self-paced or experimenter-paced option is to be used. If the experimenter-paced option is requested, you are asked to input the number of milliseconds per word for each condition. Then the following loop takes over:

\author{
repeat \\ get the current condition values \\ read and display the current stimulus \\ output results \\ until no more experimental trials or \\ input stimuli remain.
}

Although this is algorithmic, it explains the options available in our program. (This program can be obtained at a nominal cost from the author.)

\section{THE USEFULNESS OF RSVP AS A READING AID}

\section{Experimental Results Obtained by Using One-Word Stationary RSVP}

Early work with RSVP indicated that the one-word stationary technique allows readers to comprehend text at very rapid rates. For example, readers recall $70 \%$ of the words in a sentence when rates of 960 words/ min are used to present material (Forster, 1970). At slower rates ( 720 words $/ \mathrm{min})$, sentences can be recalled with $87 \%$ accuracy (Potter, Kroll, Yachzel, \& Cohen, 1978). Moreover, stationary one-word RSVP presentation of sentences is vastly superior to normal presenta. tion of sentences when both are presented for equal periods of time (Potter et al., 1978).

Studies addressing the effectiveness of RSVP for presenting paragraphs of text have indicated that at rates of 720 words/min, single-word stationary RSVP produces recall that is far more complete than that produced by normally presented paragraphs at equivalent rates. Additionally, contrasts of single-word RSVP performance at 720 words $/ \mathrm{min}$ with performance under normal presentation at a subject's preferred reading rate revealed that the two conditions produce statistically equivalent recall (Potter \& Kroll, 1977).

Unfortunately, single-word RSVP has not proved to be consistently better than normal reading at rapid rates when texts longer than a paragraph are used. Both Juola et al. (1982) and Potter et al. (1980) have shown that use of stationary RSVP input units ranging in size from one to three words produces comprehension that is roughly equivalent to that obtained from skimming large texts. The rates of presentation in these studies ranged from 214 to 720 words/min. Young et al. (1984) obtained some data that may cast some light on the failure of stationary RSVP to improve comprehension over that with normal fast reading when texts longer than a paragraph in length are presented.

\section{Experimental Results Obtained by Varying Unit \\ Size in Stationary RSVP}

In early work, we manipulated rate of input and unit size in stationary RSVP tasks. Three rates were used to present text. The fastest was the rate at which subjects could recognize words at $75 \%$ accuracy. The medium rate was the subjects' average reading rate, and the slow rate added $1,500 \mathrm{msec}$ to the subjects' normal reading rate. Unit size varied from single words to clauses to sentences. One of the more interesting results obtained from this early work was a significant interaction between rate of input and unit size. The obtained recall protocols indicated that the unit sizes associated with optimal performance varied as a function of rate of input. At normal and slow reading rates, subjects recalled significantly more text when clauses or sentences were the unit of input, as opposed to individual words (see Table 1). However, at fast rates (approximately 1,200 words $/ \mathrm{min}$ ), the single-word unit size produced the best recall.

In another series of studies, texts approximately 350 words in length were presented at slightly speeded rates (300 words/min). Unit size was also manipulated,

Table 1

Speed x Unit Size Interaction Collapsed Across Reader Groups, Idea Units-Experiment 1*

\begin{tabular}{lrrrr} 
& \multicolumn{4}{c}{ Speed of Presentation } \\
\cline { 2 - 5 } Unit Size & Fast & , Medium & Slow & Marginal \\
\hline Words & 2.1 & 7.9 & 9.5 & 6.5 \\
Clauses & 1.0 & 10.4 & 13.0 & 8.1 \\
Sentences & .8 & 9.9 & 13.8 & 8.1 \\
Marginal & 1.3 & 9.4 & 12.1 & \\
\hline
\end{tabular}

${ }^{*}$ Tukey WSD minimal significance level $=1.74, p<.05$. 
Table 2

Idea Units Recalled-Experiment 2*

\begin{tabular}{lc}
\hline \multicolumn{1}{c}{ Condition } & Number Recalled \\
\hline Total text & 8.6 \\
Words stationary & 11.8 \\
Three words stationary & 10.8 \\
Clauses stationary & 11.8 \\
\hline
\end{tabular}

${ }^{*}$ Tukey WSD minimal significance level $=0.88, p<.05$.

Table 3

Macro-Idea Units and Idea Units Collapsed Across Moving and Stationary Conditions $x$ Unit Size-Experiment 2*

\begin{tabular}{lccc}
\hline & Words & Three Words & Clauses \\
\hline Idea units & 8.9 & 9.4 & 10.1 \\
Macro-idea units & .28 & .46 & .41 \\
\hline
\end{tabular}

${ }^{*}$ Tukey WSD minimal significance level for macros $=0.12$, $p<.05 ;$ Tukey WSD minimal significance level for idea units $=$ $1.06, p<.05$.

but in these studies single words, clauses, and three-word units were the unit sizes investigated. Subjects also read a complete text that was presented in whole form, but their reading time was restricted such that they were allowed the same amount of time per word in all conditions.

The results revealed that significant comprehension gains were obtained by presenting RSVP words and clauses. These unit sizes were associated with significantly more thorough text comprehension than was normal reading (see Table 2). Furthermore, use of clausesized input units was associated with a significant increase in the number of macro-propositions recalled relative to the number recalled with normal reading and with all other RSVP unit sizes (see Table 3). The above findings may explain why Juola et al. (1982) failed to find any significant advantage of using one- and twoword unit sizes at rates under 350 words/min. Furthermore, the earlier rate $\mathrm{x}$ unit size interaction can also explain the Potter et al. (1980) finding that singleword stationary RSVP was inferior to normal reading at 240 and 480 words/min but not inferior to skimming regular texts at 720 words $/ \mathrm{min}$.

\section{THE USEFULNESS OF RSVP AS A RESEARCH TOOL}

There is some debate over the validity of RSVP as a research tool. Some investigators have maintained that RSVP is not a valid research tool because it alters the information processing requirements of reading. To the contrary, it is a most useful tool for this very reason. Specifically, by contrasting RSVP conditions in which certain component processes are hypothetically necessary or unnecessary for accurate text comprehenison and retention, we can gain insight into the operation of such components. However, to perform such contrasts, one needs complete task analyses of the conditions to be contrasted.
Just et al. (1982) compared self-paced moving and stationary one-word RSVP with the gaze-duration patterns obtained from eye movement monitoring studies. Only the moving, one-word RSVP reading times mirrored the patterns obtained from eye movement monitoring. Recall protocols were also obtained; unfortunately, the investigators failed to contrast the one-word moving and stationary results. As a result, they concluded that RSVP is an invalid research tool because the information processing requirements are different from those in normal reading.

It is necessary to interpret all empirical results in conjunction with the methodology used to obtain them. Just as it is necessary to analyze each type of RSVP implementation separately, it is also necessary to perform a task analysis on all other experimental techniques. Eye movement monitoring has been used to try to uncover some of the component processes underlying reading. Many people who have used the eye movement or gaze-duration techniques assume that gaze duration is a valid indicator of the time consumed by component processes of reading. However, if you look at the task, the amount of time devoted to eye movement execution and planning is deeply embedded in this methodology. The implicit assumption is that eye movement execution and planning are constant across all stimulus situations. As far as we know, these are untested assumptions.

Just et al. (1982) found that the reading-time patterns associated with stationary RSVP differed from normal reading patterns obtained by monitoring eye movements. Just because the patterns of time spent on single words vary depending upon whether eye movements are required or not does not imply that RSVP is an invalid research tool. In contrast, it appears that such comparisons might provide some insight into the nature of eye movements and their effect on the component processes operating in reading. It is possible that some of the time that would normally be allocated to planning and executing eye movements could be allo. cated to other tasks such as integration.

We (Young, Angell, \& Bourne, 1984) adapted the Just et al. (1982) idea of contrasting moving and stationary RSVP in order to investigate eye movements. We compared the recall protocols obtained from presenting moving and stationary RSVP text and whole pages of text. The RSVP unit sizes were single words, threeword units, and clauses. The results revealed that a general deficit was associated with all moving-window conditions. Furthermore, as unit size decreased, the differences between moving and stationary RSVP increased. For example, the one-word moving condition was most detrimental to subjects, whereas the one-word stationary condition facilitated performance (both relative to normal reading). Because the one-word stationary condition almost completely eliminates the need to perform eye movements and the one-word moving condition necessitates more eye movements 
than are normally made while reading complete texts (because not all words are read), these results suggest that the eye movement system competes for resources with the rest of the component processes. By eliminating eye movements, more resources appear to be devoted to maintaining items in memory.

RSVP can also be used to investigate many other information processing and reading comprehension issues. For example, Masson (1983), investigating integration, used single-word stationary RSVP with and without pauses inserted at the end of sentences, while manipulating rate of presentation. Masson's results indicated that inserting pauses substantially increased comprehension. Furthermore, comprehension associated with singleword stationary RSVP was equal to normal reading at 500 words/min when pauses were inserted. These results indicate the necessity of allowing time for integrative processing to operate.

In sum, the question of whether or not RSVP is a valid research tool depends on the research question being investigated. This point was made initially by Ward and Juola (1982) and was expounded upon earlier by Aaronson (1984) in this symposium. RSVP can provide substantial insight into the component processes involved in the complex information processing task of reading and text comprehension.

\section{REFERENCES}

Anrongon, D. (1984). Computer methods and ecological validity in reading research. Behavior Research Methods, Instruments, \& Computers, 16, 102-108.

Aaronson, D., \& Scarborough, H. S. (1977). Performance theories for sentence coding: Some quantitative models. Journal of Verbal Learning and Verbal Behavior, 17, 277-303.
CARr, T. H. (1981). Research on reading: Meaning, context effects and comprehension. Journal of Experimental Psychology: Human Perception and Performance, 7, 592-603.

Cocklin, T., Ward, N. J., Chen, H. C., \& Juola, J. (1983). Factors influencing readability of rapidly presented text segments. Manuscript submitted for publication.

Fonster, K. I. (1970). Visual perception of rapidly presented word sequences of varying complexity. Perception \& Psychophysics, 8 , 215-221.

JuOLA, J. F., WARd, N. J., \& McNamara, T. (1982). Visual search and reading of rapid serial presentation of letter strings, words and text. Journal of Experimental Psychology: General, 111, 208-227.

Just, M. A., \& Carpenter, P. A. (1980). A theory of reading: From eye fixations to comprehension. Psychological Review, 87, 329-354.

Just, M. A., Carpenter, P. A., \& Woolley, J. D. (1982). Paradigms and processes in reading comprehension. Journal of Experimental Psychology: General, 111, 228-238.

Masson, M. E. J. (1983). Conceptual processing of text during skimming and rapid sequential reading. Memory \& Cognition, $11,262-274$

Potten, M. C., \& Kroll, J. F. (1977, July). RSVP paragraphs (Tech. Rep. No. 4, ARPA Contract No. MDA 903-76-C-0441). Cambridge, MA: M.I.T.

Potter, M. C., Kroll, J. F., \& Harris, C. (1980). Comprehension and memory in rapid sequential reading. In $\mathbf{R}$. S. Nickerson (Ed.), Attention and performance VIII. Hillsdale, NJ: Erlbaum.

Potter, M. C., Kroll, J. F., Y achzel, B., \& Cohen, J. (1978, July). Pictures in sentences: Conceptual and lexical representations in language comprehension (Tech. Rep. No. 8, ARPA Contract No. MDA 903-76-C-0441). Cambridge, MA: M.I.T.

Wand, N. J. (1981). An evaluation of the effects of rapid, serial presentations on reading comprehension. Unpublished doctoral dissertation. University of Kansas, 1981.

WARD, N. J., \& JUOLA, J. F. (1982). Reading with and without eye movements: Reply to Just, Carpenter and Woolley. Journal of Experimental Psychology: General, 111, 239-241.

Young, S. R., Angelt, L. S., \& Bourne, L. E., Jr. (1984). Resources in reading: An interactive approach. Manuscript submitted for publication. 